\title{
LAYER FORMATION ON CLEAN SURFACES STUDIED BY PHOTOELECTRON SPECTROSCOPY
}

\author{
R. NYHOLM \\ Department of Synchrotron Radiation Research, Institute of Physics, Lund University \\ Sölvegatan 14, 22362 Lund, Sweden \\ and \\ MAX-LAB, Lund University, Box 118, 22100 Lund, Sweden
}

\begin{abstract}
A review of recent experiments on overlayer systems on single crystal metal substrates are presented. The experiments are based on high resolution photoelectron spectroscopy on adsorbate and substrate core levels utilizing synchrotron radiation from the MAX I storage ring in Lund, Sweden. The systems discussed in this paper are alkali metal overlayers on aluminum and the adsorption of molecular carbon monoxide on palladium.
\end{abstract}

PACS numbers: 73.20.At, 79.60.-i

\section{Introduction}

Core level photoelectron spectroscopy has long been used to study the electronic structure of pure and compound materials. The power of the technique lies in the observation of small shifts in the binding energy of a core level (so-called chemical shifts) when the chemical surrounding of the photoemitting atom is changed. When synchrotron radiation is used for the excitation the tunability of the photon energy allows for a very high surface sensitivity. Thus the method is well suited for the study of adsorbate systems, surface overlayers, surface compounds and interfaces.

Substantial progress in photoelectron spectroscopy for the study of surfaces has been made in recent years using tunable synchrotron radiation [1-4]. This progress is mainly due to the development of better radiation sources, such as low emittance storage rings, multi-pole wigglers, undulators, etc., and also the development of more powerful monochromators. This has lead to a wider photon energy range becoming accessible at high resolution. In some cases the inherent line widths, due mainly to lifetime broadening and phonon broadening, are now setting the limit of how small binding energy shifts can be detected and accurately determined. However, there is still a need for further development, especially in the photon energy range from about $500 \mathrm{eV}$ and above, e.g. photon energies up to $1 \mathrm{keV}$ with about $0.1 \mathrm{eV}$ resolution, and high flux, would be desirable in studies of deeper core levels such as the $2 p$ levels in the late $3 d$ transition metal series. 
The present status of monochromatic photon sources with fluxes high enough to be of use in core level photoelectron spectroscopy can be examplified by the following beamlines and monochromators: The plane grating SX-700 type of monochromators at BESSY, Berlin [5] and MAX-LAB, Lund [6], and the spherical grating monochromators at NSLS, Brookhaven $[7,8]$ and Super ACO, Orsay [9]. Very high resolving powers $(E / \Delta E)$ of up to about $10000 \mathrm{~atm}$ several hundred $\mathrm{eV}$ have been reported [8]. However, one has to bear in mind that in most cases these extreme resolving powers are obtained at the expense of photon flux. Under normal experimental conditions and when large data sets have to be collected the resolving power has to be reduced for practical reasons. In this context high brilliance photon sources and high performance optical designs are crucial in order to maintain the necessary photon flux at the small slit settings and/or the small angular acceptances needed for high resolution. Equally important is of course to improve the efficiency of present day electron energy analyzers.

In this paper some recent results from studies of overlayer systems using high resolution photoelectron spectroscopy will be presented. All data shown have been obtained at beamline $22 \mathrm{~atm}$ the MAX-laboratory in Lund [6]. This beamline is equipped with a modified SX-700 type of plane grating monochromator and an experimental station with a large hemispherical electron energy analyzer. In the experiments presented here the resolving power of the monochromator ranged from about $5000 \mathrm{~atm} 100 \mathrm{eV}$ to about $2000 \mathrm{~atm} 400 \mathrm{eV}$. The hemispherical electron energy analyzer has a mean radius of $200 \mathrm{~mm}$ and is equipped with a multichannel detector system which makes it possible to record spectra at energy resolutions of 20 to $30 \mathrm{meV}$ with high efficiency. Typical recording times for the spectra presented in this paper were 10 to 20 minutes.

\section{Results and discussion}

\subsection{General}

The examples shown in this paper cover different systems such as clean surfaces, non-reactive and reactive overlayers and molecular adsorption systems. The core level spectra are treated in different ways depending on the complexity of the system. It is therefore appropriate to give a short summary of possible ways to analyze core level spectra.

Core level binding energy shifts can be treated on different levels of sophistication as will be shown in the following examples. The mere observation of a core level binding energy shift is an indication that the chemical environment of the photoemitting atom has undergone a change. For instance in a system where an overlayer of one material is deposited onto a clean substrate the observation of shifted core level peaks in the substrate and/or the overlayer gives information on the interaction in the surface layer. Examples will be given of systems where the overlayer grows on top of the substrate without mixing as well as systems where an intermixing between the substrate and the deposited material occurs. In both cases the appearance of different components in the substrate and overlayer core level spectra yield a straightforward interpretation of the general behavior in the surface layer. 
The next level of sophistication involves an understanding of the magnitude of the core level shifts. In some of the examples shown in this paper a model connecting core level binding energy shifts to thermochemical quantities, such as surface energies, segregation energies and adhesion energies, will be used. This model can be used either to extract thermochemical quantities from observed binding energy shifts or to calculate core level binding energy shifts from the known thermochemical data and thereby facilitate the identification of different core level peaks in measured spectra. The basic assumption in this model [10] is that the core ionized final state is completely screened by the valence electrons, which has been shown to be an accurate assumption for metallic systems. With this screening in mind one may regard the core ionized atom as a $Z+1$ impurity in an otherwise unperturbed $Z$ metal. Since the core level binding energy is the difference in total energy between the core ionized final state system (a $Z+1$ impurity in a $Z$ metal) and the ground state system (the $Z$ metal) it is fairly straightforward to obtain relations between core level binding energy shifts and thermochemical quantities related to a $Z+1$ impurity in a $Z$ metal.

The most sophisticated way of treating core level binding energy shifts is to compare the measured shifts to first principal calculations. Such calculations, based on the total energy difference between the core ionized state and the ground state, are rather scarce, but hopefully developments in computational techniques will make these kinds of calculations more readily available in the near future. Especially appealing is the possibility to calculate binding energy shifts for different model geometrical structures, which when compared to measured shifts would make geometrical structure determinations by core level photoelectron spectroscopy more accessible. As will be shown below first principle calculations are able to predict accurately the surface core level shift for the $\mathrm{Al}(100)$ surface.

\subsection{The Al(100) and Al(111) surfaces}

The surface core level shifts of different crystal faces of aluminum have been the subject of several investigations in the past [11-13]. The most studied surface is (100) for which differences in the spectral shape have been observed between bulk and surface sensitive spectra in both photoemission [11,13] and photoabsorption [12] measurements on the $\mathrm{Al} 2 p$ core level. The broadenings that were seen in the surface sensitive spectra were interpreted as due to an enhanced crystal field splitting for the surface atoms and/or due to a surface core level shift. Values for the surface core level shift ranging from $-40 \mathrm{meV}$ to $-120 \mathrm{meV}$ have been reported. The reason for this wide spread in the reported surface shift was due to the fact that the shift is too small to be accurately determined in comparison to the combined inherent line width of the $2 p$ level and the experimental resolution obtainable in the experiments. For the (111) surface no difference between surface and bulk sensitive spectra have been observed and the earlier experiments are consistent with an exceedingly small shift (less than $50 \mathrm{meV}$ [13]) if any at all.

Since these experiments in the early eighties the development of both monochromatic light sources and electron energy analyzers has made it possible to resolve the experimental uncertainty for the surface core level shift of the (100) 
surface and to some extent also for the (111) surface [14]. The Al $2 p$ core level with a binding energy of $73 \mathrm{eV}$ can be studied using photon energies around $100 \mathrm{eV}$ where a photon energy resolution of about $20 \mathrm{meV}$ can be reached. Together with the high efficiency electron energy analyzer described above a total energy resolution of $50 \mathrm{meV}$ was used in the experiments. At this high experimental resolution the Al $2 p$ spectra from the (100) surface shows a clear surface shift as seen in Fig. 1. Both spin-orbit components (spin-orbit split of $0.46 \mathrm{eV}$ ) have a clear shoulder to

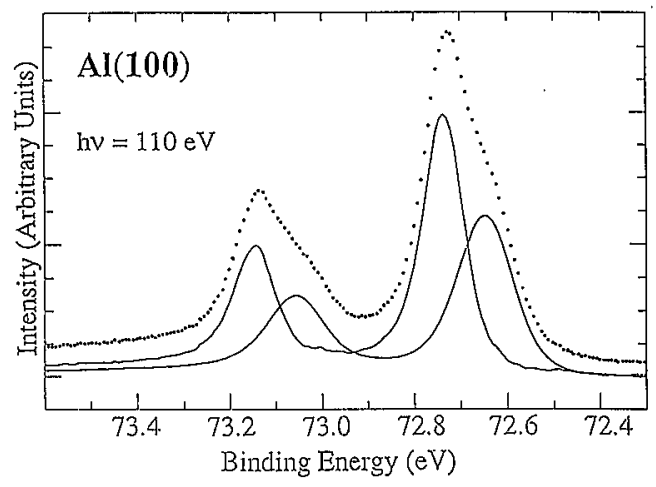

Fig. 1. The $2 p$ core level spectrum from $\mathrm{Al}(100)$ (dots) and a decomposition into bulk and surface components (solid curves). From Ref. [14].

lower binding energy. From the relative intensity changes as a function of the photon energy the low binding energy structure is identified as due to emission from the surface atoms. The two spin-orbit components are very similar with no sign that the surface related $2 p_{3 / 2}$ component is broader than the $2 p_{1 / 2}$ component which would be expected if crystal field splitting was dominant (only the $2 p_{3 / 2}$ component is expected to be effected by crystal-field splitting). Thus already the raw spectra of Fig. 1 clearly show the existence of a surface core level shift towards lower binding energy. A more detailed analysis involving a curve fitting procedure gives a numerical value of $-96 \mathrm{meV}$ for the surface shift.

The Al(111) spectrum shown in Fig. 2 is completely different from that of the (100) surface. There is no sign of any extra structure in the two spin-orbit components and any surface shift must be very small. A more quantitative support for this conclusion is obtained by comparing the bulk part of the spectrum from the (100) surface to the spectrum from the (111) surface (see Fig. 2). The bulk (100) spectrum and the total (111) spectrum are almost identical. If the (111) spectrum is modelled using the bulk and surface line profiles deduced from the (100) spectrum one obtains an upper limit for the $\mathrm{Al}(111)$ surface shift of $15 \mathrm{meV}$.

As mentioned above core level binding energies may be treated within the $Z+1$ model which is also applicable in describing surface core level shifts. According to the model of Johansson and Mårtensson $[10,15]$ the surface core level - binding energy shift can be related to the difference in surface energies $\left(\gamma_{\mathbf{s}}\right)$ of the $Z+1$ metal (representing the core ionized final state) and the $Z$ metal with a 


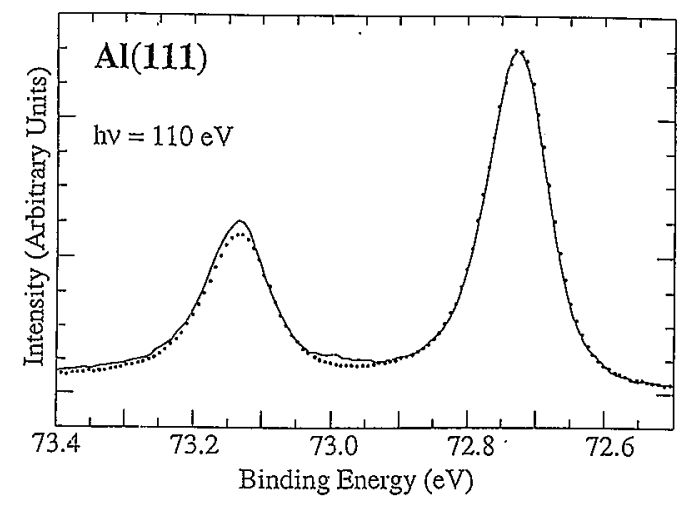

Fig. 2. Al $2 p$ spectrum from the $\mathrm{Al}(111)$ surface (dots). The bulk spectrum decomposed from the $\mathrm{Al}(100)$ spectrum (solid line) is also shown. From Ref. [14].

small correction involving impurity energies of a $Z+1$ atom in the bulk and at the surface of the $Z$ metal:

$$
\Delta E_{\text {surf }}=\gamma_{\mathbf{s}}(Z+1)-\gamma_{\mathbf{s}}(Z)-\left[E_{\text {imp }}^{\text {bulk }}(Z+1, Z)-E_{\text {imp }}^{\text {surf }}(Z+1, Z)\right] .
$$

Applying this formula to the $\mathrm{Al}(100)$ surface yields a shift of $-106 \mathrm{meV}$ [13] in agreement with the experiment. For the (111) surface a shift close to zero is obtained [13].

More interesting, however, is that for the $\mathrm{Al}(100)$ surface $a b$ initio calculations for the surface shift has been made [16]. These calculations give a surface shift of $-97 \mathrm{meV}$ in excellent agreement with the experimental value. The surface core level shift is obtained by calculating the total energy difference between a system with the core ionized atom at the surface and in the bulk, respectively, thereby taking full account of final state relaxation effects. Furthermore, the core ionized final state atom is treated as a Si impurity, showing that the $Z+1$ model correctly describes the final state core ionized atom. So far no corresponding calculations have been made for the (111) surface.

\subsection{The Pd(100) surface}

Very few measurements have been made for the $4 d$ transition metal series (see e.g. Ref. [3]). The only narrow core level in these elements (the $3 d$ level) has rather high binding energies which makes high resolution measurement difficult.

Figure 3 shows $3 d$ core level spectra from the $\mathrm{Pd}(100)$ surface recorded at photon energies ranging from $380 \mathrm{eV}$ to $450 \mathrm{eV}$ [17]. The total experimental resolution was in the range from 0.2 to $0.3 \mathrm{eV}$ which allows for a separation between surface and bulk emission. In the series of spectra recorded at different photon energies both the $3 d_{3 / 2}$ and $3 d_{5 / 2}$ spin-orbit components (spin-orbit split of $5.3 \mathrm{eV}$ ) show a distinct shoulder towards lower binding energy. From the relative intensity changes of this low binding energy component as a function of excitation energy it can be identified as due to emission from surface atoms. A decomposition of these 


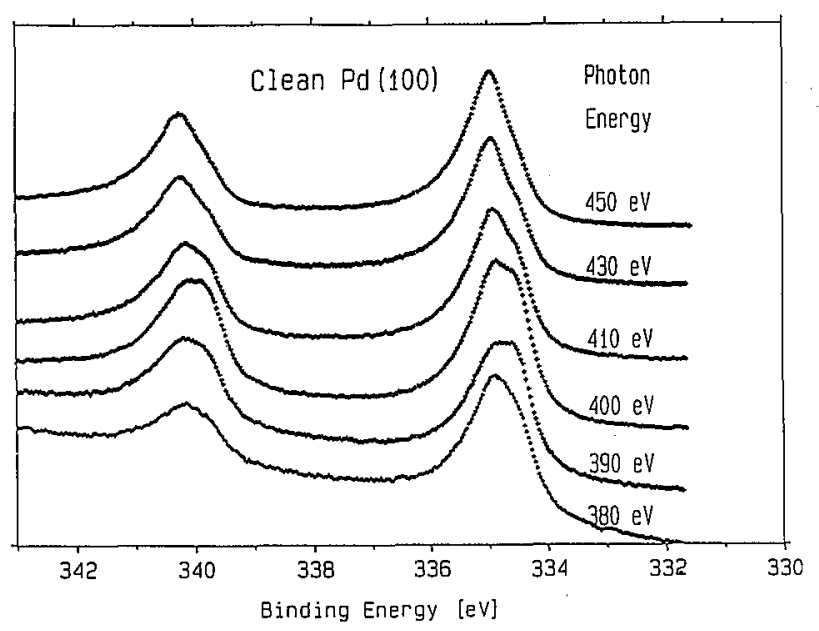

Fig. 3. Pd $3 d$ core level spectra from $\mathrm{Pd}(100)$ recorded at different photon energies. From Ref: [17].

spectra into bulk and surface contributions, which can be done either by a curve fitting procedure or by subtraction procedures, gives a surface core level shift of $-0.44 \pm 0.03 \mathrm{eV}[17]$.

The calculated surface core level shift using Eq. (1) gives values in the range from $-0.27 \mathrm{eV}$ to $-0.40 \mathrm{eV}$. The reason for this spread is simply due to the fact that the surface energies of $\mathrm{Pd}$ and $\mathrm{Ag}$ (the $Z+1$ element) are not accurately known. For Ag experimental values and theoretical calculations are consistent, but for Pd no experimental values are available and different theoretical values are not in complete agreement (see discussion in Ref. [17]). The last term in Eq. (1) can be estimated to be only about $0.05 \mathrm{eV}$.

\subsection{Alkali overlayers on Al(111)}

Depositing alkali metals onto an $\mathrm{Al}(111)$ surface at $100 \mathrm{~K}$ results in an unreacted overlayer. Following the growth from submonolayer coverage to multilayer coverage the alkali core level spectra change dramatically. This is due to different core level binding energies for the interface, bulk and surface atoms in the alkali overlayer. Figure 4 shows alkali core level spectra from three layers of $\mathrm{Na}, \mathrm{K}, \mathrm{Rb}$ and Cs deposited on $\mathrm{Al}(111)$ [18]. All spectra exhibit a number of different components, the $\mathrm{Na} 2 p$ and $\mathrm{K} 3 p$ spectra having a more complicated structure due to the small spin-orbit splitting ( $0.16 \mathrm{eV}$ and $0.26 \mathrm{eV}$, respectively). An identification of the different components, as indicated in the figure, can easily be done by following the gradual appearance of structures as a function of layer thickness. Thus the low binding energy component is due to emission from the atoms at the interface to the $\mathrm{Al}(111)$ substrate, the high binding energy component comes from the surface atoms and the middle component is due to the intermediate layers, denoted as bulk atoms. Another observation, which is clearly made from the development of the 


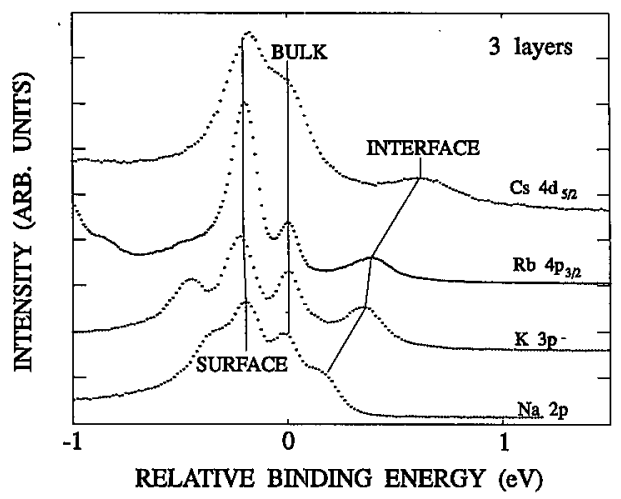

Fig. 4. Alkali core level spectra from about three layer depositions on $\mathrm{Al}(111)$ at $100 \mathrm{~K}$. The spectra are shown on a relative binding energy scale with the bulk peaks aligned to zero eV. From Ref. [18].

spectra as a function of layer thickness, is that the alkali overlayer does not grow in a layer by layer fashion, e.g. the third layer starts to grow before the second layer is completed.

\section{TABLE}

Experimental and calculated binding energy shifts (in $\mathrm{eV}$ ) for different alkali overlayers on $\mathrm{Al}(111)$. For details see text. Data from Ref. [18].

\begin{tabular}{c|c|c|c|c}
\hline \hline & \multicolumn{2}{|c|}{$\Delta E_{\text {adh }}$} & \multicolumn{2}{c}{$\Delta E_{\text {int }}$} \\
\cline { 2 - 5 } & Exp. & Calc. & Exp. & Calc. \\
\hline $\mathrm{Na} / \mathrm{Al}$ & -0.37 & -0.36 & -0.18 & -0.24 \\
$\mathrm{~K} / \mathrm{Al}$ & -0.56 & -0.81 & -0.36 & -0.69 \\
$\mathrm{Rb} / \mathrm{Al}$ & -0.59 & -0.86 & -0.39 & -0.70 \\
$\mathrm{Cs} / \mathrm{Al}$ & -0.82 & -0.92 & -0.62 & -0.76 \\
$\mathrm{Al} / \mathrm{Na}$ & -0.07 & -0.03 & -0.07 & -0.04 \\
$\mathrm{Al} / \mathrm{K}$ & -0.08 & -0.04 & -0.08 & -0.05 \\
$\mathrm{Al} / \mathrm{Rb}$ & -0.10 & -0.04 & -0.10 & -0.05 \\
$\mathrm{Al} / \mathrm{Cs}$ & -0.14 & -0.04 & -0.14 & -0.06
\end{tabular}

Turning now to the binding energy shifts we observe that for all alkalis the surface shift is about equal. The magnitude of these surface shifts are also in very good agreement with values calculated from Eq. (1). The shift between the bulk 
peak (or the surface peak) and the interface peak increases with increasing atomic number of the alkali metal. The experimental shifts are summarized in Table.

The $\mathrm{Al} 2 p$ spectra from the alkali covered $\mathrm{Al}(111)$ substrate are shown in Fig. 5. In all cases one sees, in addition to the bulk peak, a small component

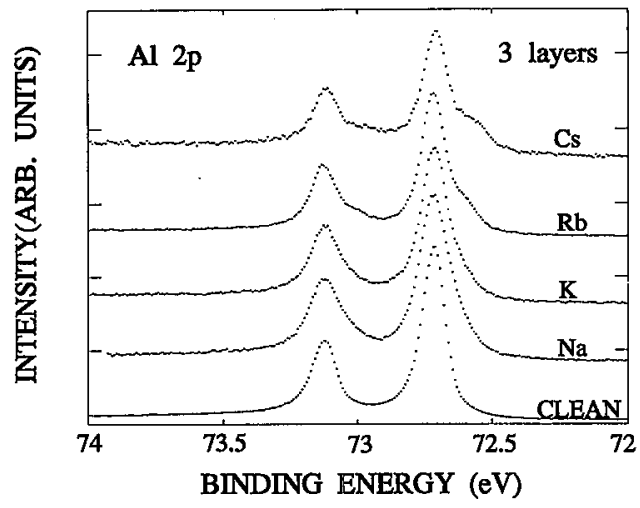

Fig. 5. Al $2 p$ spectra from the same alkali overlayer systems as in Fig. 4. From Ref. [18].

shifted to lower binding energies. The shift of this interface component also shows an increasing trend with increasing atomic number of the alkali metal. The experimental shifts are summarized in Table.

A further analysis of the core level shifts can be made within the $Z+1$ model in the following way. First let us define the shift between the interface and the surface peaks as an adhesion shift $\Delta E_{\text {adh }}$ and the shift between the interface and the bulk peak as an interface shift $\Delta E_{\text {int }}$. The reason for these definitions become clear from the schematic picture in Fig. 6 . Here the core level binding energies are schematically shown as the difference in total energy between the

$$
\begin{aligned}
& { }^{\mathrm{a} /} \Delta \mathrm{E}_{\mathrm{lnt}}=[\boxminus-\boxminus]-[\boxminus-\square]=\square-\boldsymbol{\square} \\
& { }^{\mathrm{b}} \Delta \mathrm{E}_{\mathrm{Adh}}=[\varpi-\boxminus]-[\square-9]= \\
& =[\boldsymbol{\theta}-\boldsymbol{g}]-[\boxminus-g] \approx \boldsymbol{E}_{\mathrm{Adh}}^{\mathrm{Z+1,M}}-\boldsymbol{\varepsilon}_{\mathrm{Adh}}^{\mathrm{ZM}}
\end{aligned}
$$

Fig. 6. Schematic representation of the connection between segregation and adhesion energies and the binding energy shift between the interface and bulk peak $\Delta E_{\text {int }}$ and the interface and surface peak $\Delta E_{\mathrm{adh}}$, respectively. The shaded boxes represent the substrate, the open boxes the overlayer and the filled small boxes represent a core ionized atom treated as a $Z+1$ impurity (see also text). $\varepsilon_{\text {adh }}$ are adhesion energies per atom. From Ref. [18] and [19]. 
core ionized final state and the ground state. Furthermore, the core ionized final state is approximated as a $Z+1$ impurity in the otherwise unchanged $Z$ metal. For instance, for the adhesion shift $\Delta E_{\text {adh }}$, the first bracket gives the core level binding energy of the interface atoms and the second bracket gives the core level binding energy of the surface atoms. Rearranging the symbols it becomes clear that the adhesion shift $\Delta E_{\text {adh }}$ is nothing but the difference in adhesion energies for a $Z+1$ metal and a $Z$ metal to an $M$ metal substrate. In the same way the interface shift $\Delta E_{\text {int }}$ is the segregation energy of a $Z+1$ impurity in a $Z$ metal to the $Z / M$ interface, i.e. an interface segregation energy. This model, relating core level binding energy shifts to thermochemical quantities, was first introduced by Mårtensson et al. in an investigation of $\mathrm{Yb}$ overlayers on $\mathrm{Mo}(110)$ [19].

From the experimental shifts (see Table) one may conclude that $Z+1$ impurities in the alkali layer will segregate to the alkali/Al interface, and that the interface segregation energy increases when going from $\mathrm{Na}$ to $\mathrm{K}$ to $\mathrm{Rb}$ to $\mathrm{Cs}$. From the Al $2 p$ shifts one finds that an Si impurity atom (the $Z+1$ element) will segregate to the interface but with a rather small segregation energy. In general these measurements show that thermochemical quantities like adhesion and segregation energies may be determined in a straightforward way from core level binding energy shifts. Such thermochemical quantities are most difficult to measure accurately with other experimental techniques.

If accurate experimental data for the adhesion and segregation energies were available, one could test the accuracy of the model in Fig. 6. However, this is not the case and we have to rely on calculated energies. for comparison with the measured shifts. Gerkema and Miedema [20] have devised a simple semi-empirical model to calculate adhesion and interface segregation energies. Using this model and the scheme in Fig. 6 the shifts $\Delta E_{\text {adh }}$ and $\Delta E_{\text {int }}$ can be calculated. These calculated values are given in the table and are generally found to be in good agreement with the measured data.

\section{5. $\mathrm{Na}$ on $\mathrm{Al}(111)$}

The adsorption of alkali metals on aluminum has in the past been viewed as a model adsorption system where the alkali metal atoms reside on the surface (see e.g. Ref. [21]). That this is not always the case, was recently shown by Schmalz et al. [22] for the $(\sqrt{3} \times \sqrt{3}) \mathrm{R} 30^{\circ}$ structure formed by adsorbing $1 / 3$ of a monolayer of $\mathrm{Na}$ on $\mathrm{Al}(111)$. Using polarization dependent surface extended X-ray absorption fine structure (SEXAFS) measurements they concluded that $\mathrm{Na}$ occupies a sixfold coordinate site formed by displacing every third $\mathrm{Al}$ atom in the $\mathrm{Al}$ surface layer. This adsorption structure was also shown to be energetically favorable by first principal calculations [22]. Later J.N. Andersen et al. [23] investigated several ordered overlayer structures of $\mathrm{Na}$ on $\mathrm{Al}(111)$ using core level photoelectron spectroscopy, and found that intermixing between $\mathrm{Na}$ and $\mathrm{Al}$ is far from unusual. In the following we will review these later measurements and see how photoelectron spectroscopy can be used to investigate surface compounds.

$\mathrm{Na}$ adsorbs in several well-ordered structures on the closed packed $\mathrm{Al}(111)$ surface as observed by low energy electron diffraction (LEED). At room temperature two ordered structures occur; a $(\sqrt{3} \times \sqrt{3}) \mathrm{R} 30^{\circ}$ structure at one third of 
a monolayer coverage and at saturation coverage a $2 \times 2$ structure. If the $2 \times 2$ structure is cooled to $150 \mathrm{~K}$ and more Na is deposited a $(2 \sqrt{3} \times 2 \sqrt{3}) \mathrm{R} 30^{\circ}$ structure will form. The $\mathrm{Al} 2 p$ spectra from these three overlayer structures are shown in Fig. 7 together with the spectrum from the clean surface, a surface covered by one layer of $\mathrm{Na}$ (deposited at $100 \mathrm{~K}$ ), and a sample prepared by evaporating $\mathrm{Al}$ on a thick $\mathrm{Na}$ film. The $\mathrm{Na} 2 p$ spectra from the three ordered overlayer structures

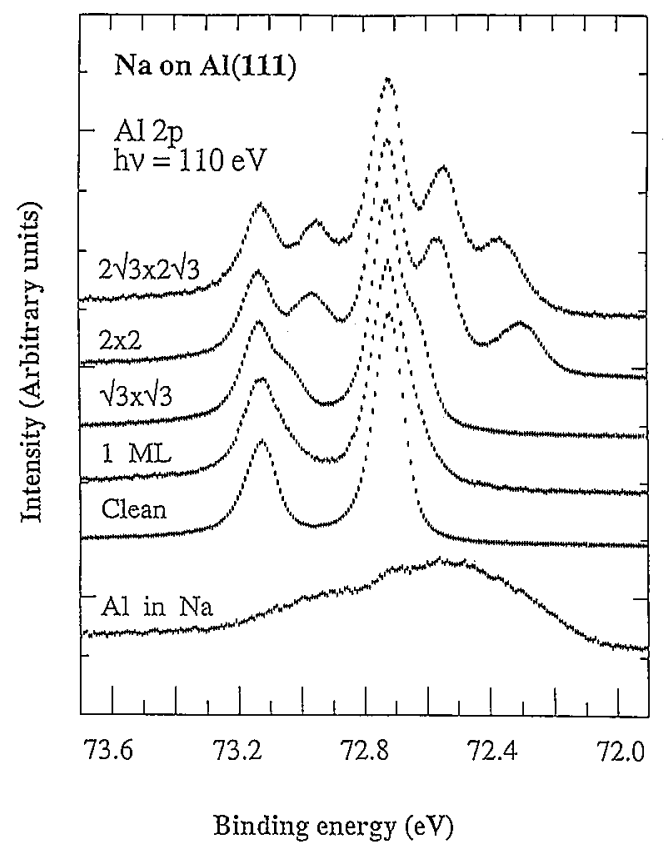

Fig. 7. Al $2 p$ core level spectra from the clean $\mathrm{Al}(111)$ surface and from different $\mathrm{Na}$ overlayer structures. The $\mathrm{Al}$ in $\mathrm{Na}$ spectrum is from a sample prepared by depositing Al on a thick Na film. From Ref. [23].

are shown in Fig. 8, together with spectra from a single layer and a multilayer deposited at $100 \mathrm{~K}$.

The $\mathrm{Al} 2 p$ spectrum from the clean surface (as discussed in connection with Fig. 2) shows the $2 p_{1 / 2}$ and $2 p_{3 / 2}$ spin-orbit components with no sign of any surface shifted components. When looking at the spectra from the $\mathrm{Na}$ overlayers we see that the most striking changes occur for the $2 \times 2$ and $(2 \sqrt{3} \times 2 \sqrt{3}) \mathrm{R} 30^{\circ}$ structures where two distinctly shifted components appear. Turning to the $\mathrm{Na}$ $2 p$ spectra in Fig. 8 , it is clear that the $2 \times 2$ and $(2 \sqrt{3} \times 2 \sqrt{3}) \mathrm{R} 30^{\circ}$ structures contain two (or more) clearly shifted $2 p$ components (note that the spin-orbit components are not resolved). These observations immediately tell us that in the $2 \times 2$ and $(2 \sqrt{3} \times 2 \sqrt{3}) \mathrm{R} 30^{\circ}$ structures an intermixing between $\mathrm{Na}$ and $\mathrm{Al}$ occurs. The motivation is simply as follows. From the $1 \mathrm{ML}$ spectrum, where one layer of $\mathrm{Na}$ is deposited on the top of the Al surface, only a slight broadening of the Al $2 p$ peak towards lower binding energy is observed. The Na induced shift can 


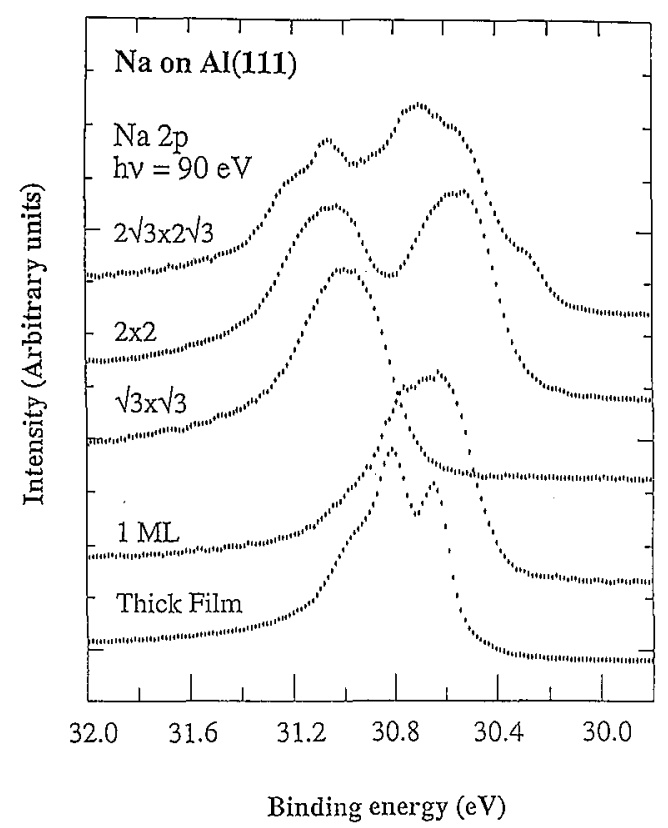

Fig. 8. Na $2 p$ core level spectra from a thick $\mathrm{Na}$ film and from different $\mathrm{Na}$ overlayer structures on $\mathrm{Al}(111)$. From Ref. [23].

be estimated to be less than $-100 \mathrm{meV}$. Thus, any larger shifts have to involve a higher coordination of $\mathrm{Al}$ to $\mathrm{Na}$. The lower spectrum in Fig. 7 recorded from a sample prepared by depositing $\mathrm{Al}$ on a thick $\mathrm{Na}$ film at $100 \mathrm{~K}$ gives further support for this conclusion. In this sample $\mathrm{Al}$ is incorporated in the $\mathrm{Na}$ film in a disordered way. A number of different $\mathrm{Al}$ to $\mathrm{Na}$ coordinations are present resulting in a very broad Al $2 p$ spectrum. However, the important observation is the appearance of components shifted by as much as $-400 \mathrm{meV}$ from the bulk $\mathrm{Al} 2 p$ peak of pure Al. From this it becomes clear that the low binding energy component in the $2 \times 2$ and $(2 \sqrt{3} \times 2 \sqrt{3}) \mathrm{R} 30^{\circ}$ structures must come from Al almost surrounded by $\mathrm{Na}$. The existence of two shifted peaks also shows that $\mathrm{Al}$ atoms with two different coordinations to $\mathrm{Na}$ are present. It seems reasonable to assign the peak with the smallest shift to $\mathrm{Al}$ atoms in the interface between the overlayer and the $\mathrm{Al}$ crystal. In a similar way the $\mathrm{Na} 2 p$ spectra from the $2 \times 2$ and $(2 \sqrt{3} \times 2 \sqrt{3}) \mathrm{R} 30^{\circ}$ structures show that both structures contain more than one $\mathrm{Na}$ layer.

Earlier LEED studies have suggested that the $2 \times 2$ structure consists of a double Na layer [24]. The present data are consistent with such a double layer with the important addition that $\mathrm{Al}$ atoms must be incorporated in this double layer of $\mathrm{Na}$.

The $(2 \sqrt{3} \times 2 \sqrt{3}) \mathrm{R} 30^{\circ}$ structure is much more complicated than the $2 \times 2$. For instance the Na $2 p$ spectra contain at least three different components showing an even higher coordination to Al than in the $2 \times 2$ structure. Thus the 
$(2 \sqrt{3} \times 2 \sqrt{3}) \mathrm{R} 30^{\circ}$ structure is not simply formed by adsorbing a Na layer on the top of an undistorted $2 \times 2$ structure. More detailed conclusions regarding this structure cannot be drawn from the photoemission data.

As mentioned above SEXAFS measurements [22] have shown that the $(\sqrt{3} \times \sqrt{3}) \mathrm{R}^{\circ} 0^{\circ}$ structure is formed by Na replacing every third Al surface atom. The photoelectron spectra in Figs. 7 and 8 are consistent with this picture. In the $\mathrm{Al} 2 p$ spectrum a small shoulder shifted to lower binding energy appears. The shift is slightly larger than for the $1 \mathrm{ML}$ case where the $\mathrm{Na}$ atoms are residing on the surface. In the $\mathrm{Na} 2 p$ spectrum a single $2 p_{1 / 2,3 / 2}$ component is seen which would be expected from the proposed structural model. The lower binding energy in the $1 \mathrm{ML}$ situation is consistent with the known trend of decreasing binding energy with increasing coverage of alkali metals.

\section{6. $C O$ on $P d(100)$}

In this example we will show how adsorbate induced core level shifts can be related to the local geometrical structure of the adsorbate layer. The system that will be studied is $\mathrm{CO}$ adsorbed on a $\operatorname{Pd}(100)$ surface [25]. For this systems three ordered overlayer structures may be formed: $p(2 \sqrt{2} \times \sqrt{2}) \mathrm{R} 45^{\circ}, p(3 \sqrt{2} \times \sqrt{2}) \mathrm{R} 45^{\circ}$ and $p(4 \sqrt{2} \times \sqrt{2}) \mathrm{R} 45^{\circ}$ at coverages of $0.5,0.67$ and 0.75 monolayers, respectively. These overlayer structures have been studied by LEED [26,27] and electron energy loss spectroscopy [28] from which geometrical models have been proposed. In all three structures the $\mathrm{CO}$ molecules are placed in bridge sites [26, 28], but the coordination number between surface $\mathrm{Pd}$ atoms and $\mathrm{CO}$ molecules changes with coverage.

Figure 9 shows the $\mathrm{Pd} 3 d_{5 / 2}$ and $\mathrm{C} 1 s$ core level spectra from the three overlayer structures together with a spectrum from the clean Pd surface. As already discussed the clean $\mathrm{Pd}(100)$ surface exhibits a surface core level shift of $0.44 \mathrm{eV}$ to lower binding energy. When $\mathrm{CO}$ is adsorbed we see that this surface peak disappears and emission on the high binding energy side of the bulk peak appears. Increasing the $\mathrm{CO}$ coverage leads to increased intensity at higher binding energies and a splitting into two components. In contrast to the large changes in the $\mathrm{Pd} 3 d$ spectra the $\mathrm{C} 1 s$ spectra remain almost unchanged. Only a small increase in line width is observed with increasing $\mathrm{CO}$ coverage.

Figure 10 shows the $\mathrm{Pd} 3 d_{5 / 2}$ spectra in more detail together with decompositions into individual components. For the $p(2 \sqrt{2} \times \sqrt{2}) \mathrm{R} 45^{\circ}$ structure one component shifted to about $0.5 \mathrm{eV}$ higher binding energy relative to the bulk peak is seen. For the two higher coverages, $p(3 \sqrt{2} \times \sqrt{2}) \mathrm{R} 45^{\circ}$ and $p(4 \sqrt{2} \times \sqrt{2}) \mathrm{R} 45^{\circ}$, a second component appears at about $1 \mathrm{eV}$ higher binding energy than the bulk peak.

In order to understand these core level binding energy shifts it is instructive to consider the structural models $[26,28]$ shown as insets in Fig. 10. For the $p(2 \sqrt{2} \times \sqrt{2}) \mathrm{R} 45^{\circ}$ structure it is seen that all Pd surface atoms are bonded to one CO molecule, we denote these type-1 surface atoms. For the $p(3 \sqrt{2} \times \sqrt{2}) \mathrm{R} 45^{\circ}$ and $p(4 \sqrt{2} \times \sqrt{2}) \mathrm{R} 45^{\circ}$ structures some of the Pd surface atoms are bonded to two CO molecules (denoted type-2 surface atoms) while the remaining surface atoms are 


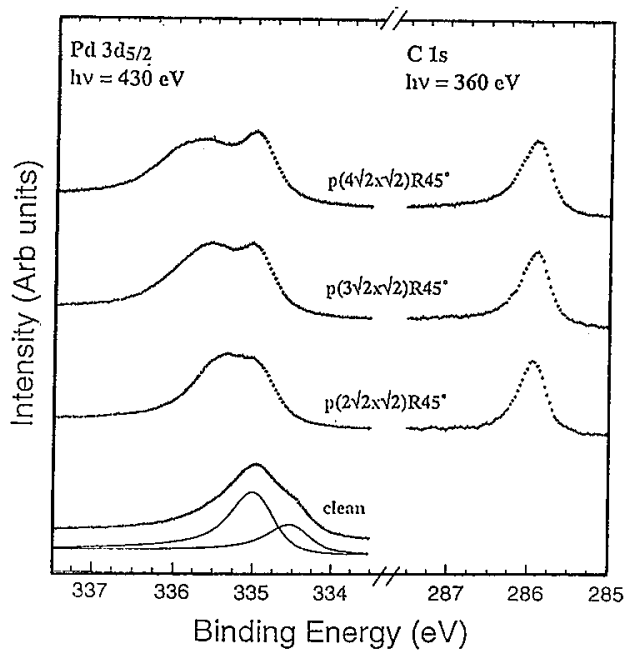

Fig. 9. $\mathrm{Pd} 3 d_{5 / 2}$ and $\mathrm{C} 1 s$ core level spectra from clean $\operatorname{Pd}(100)$ and from three different $\mathrm{CO}$ adsorption structures. For the clean Pd spectrum a decomposition into bulk (high binding energy) and surface (low binding energy) components is indicated. From Ref. [25].

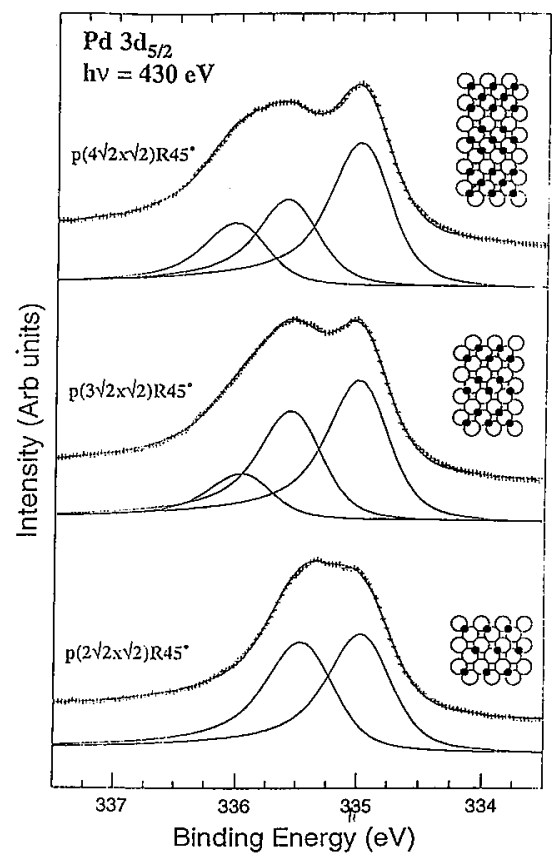

Fig. 10. Decompositions of the $\mathrm{Pd} 3 d_{5 / 2}$ core level spectra into bulk surface contributions. The insets show the structural models $[26,28]$ for the three different $\mathrm{CO}$ adsorption structures; open circles, Pd atoms; solid circles, CO molecules. From Ref. [25]. 
of type-1. The relative number of type-1 to type-2 surface atoms changes from 2:1 for the $p(3 \sqrt{2} \times \sqrt{2}) \mathrm{R} 45^{\circ}$ structure to $1: 1$ for the $p(4 \sqrt{2} \times \sqrt{2}) \mathrm{R} 45^{\circ}$ structure. This explains the observed shifts in the Pd $3 d_{5 / 2}$ spectra: For the $p(2 \sqrt{2} \times \sqrt{2}) \mathrm{R} 45^{\circ}$ structure one single shifted component is observed since all surface atoms are of type-1. In the two other overlayer structures the existence of type-2 surface atoms, bonded to two $\mathrm{CO}$ molecules, leads to the appearance of a second component shifted to higher binding energy.

The relative intensities of the two shifted components seen for the $p(3 \sqrt{2} \times$ $\sqrt{2}) \mathrm{R} 45^{\circ}$ and the $p(4 \sqrt{2} \times \sqrt{2}) \mathrm{R} 45^{\circ}$ structures are close to the relative number of type- 1 and type-2 surface atoms. The ratios are not exactly $2: 1$ and 1:1, respectively, which would be expected from the structural models. However, there could be several reasons for this deviation. For instance, because of the different local geometries of the two types of $\mathrm{Pd}$ atoms the elastic and inelastic scattering of the emitted photoelectrons may well be different for type-1 and type-2 atoms. Intuitively one would expect a larger reduction in intensity from type-2 atoms because of the higher density of $\mathrm{CO}$ molecules. Also photoelectron diffraction effects might have an influence on the relative intensities.

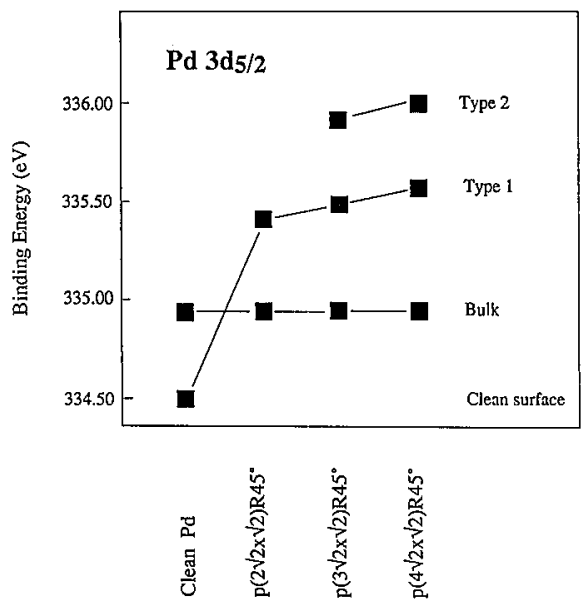

Fig. 11. Summary of the $\mathrm{Pd} 3 d_{5 / 2}$ core level binding energies for the CO/Pd(100) system. Type-1 and type-2 refer to Pd surface atoms bonded to one and two CO molecules, respectively. Data from Ref. [25].

In Fig. 11 the measured core level shifts are summarized. As seen the shift in the $3 d_{5 / 2}$ binding energy, relative to the bulk, is close to $0.5 \mathrm{eV}$ per CO-Pd bond. It is also observed that both for type-1 and type-2 atoms there is a slight increase in binding energy with increasing $\mathrm{CO}$ coverage. These small shifts reflect the fact that the type-1 and type-2 surface atoms of the different structures are not completely identical when going beyond the nearest neighbors.

Using the same basic ideas as underlying Eq. (1) the magnitude of the substrate core level shift may be related to the difference in adsorption energy of $\mathrm{CO}$ 
on the $Z$ and $Z+1$ elements, respectively (see e.g. Ref. [2]). In the present case the large shifts observed for the $\mathrm{Pd} 3 d$ core level are due to the large difference in the heat of adsorption of $\mathrm{CO}$ on $\mathrm{Pd}$ [27] and on $\mathrm{Ag}$ (the $Z+1$ element).

We should also comment on the constancy of the $\mathrm{C} 1 s$ binding energy for all three overlayer structures. In measurements on $\mathrm{CO}$ adsorbed on $\mathrm{Ni}(100)$ [29] it was found that different adsorption sites resulted in relatively large binding energy differences for the adsorbate; e.g. a $0.8 \mathrm{eV}$ shift in $\mathrm{C} 1 \mathrm{~s}$ core level was observed between on-top and bridge adsorption. In the present case the $\mathrm{C} 1 \mathrm{~s}$ binding energy stays constant within $50 \mathrm{meV}$, which is consistent with only one adsorption site for all three overlayer structures.

\section{Summary}

Examples have been given on the use of high resolution photoelectron spectroscopy to study overlayers and adsorbates on clean surfaces. It is shown that core level binding energy shifts may provide information on the local geometry in overlayer systems. In multilayer systems, like the alkali on $\mathrm{Al}(111)$ systems discussed in this paper, core level binding energy shifts between surface, bulk and interface atoms can be resolved. Within the $Z+1$ model these shifts may be related to surface energies, segregation energies and adhesion energies. Core level photoelectron spectroscopy may also be used to study intermixing between adsorbate and substrate as shown for the room temperature adsorption of $\mathrm{Na}$ on $\mathrm{Al}(111)$. Finally it was shown that the core level shifts observed in the $\mathrm{CO}$ on $\mathrm{Pd}(100)$ system can be used as a probe for the local overlayer structure.

\section{Acknowledgement}

This work is financially supported by the Swedish Natural Science Research Council.

\section{References}

[1] D. Spanjaard, C. Guillot, M.C. Desjonquères, G. Tréglia, J. Lecante, Surf. Sci. Rep. 5, 1 (1985).

[2] W.F. Egelhoff, Jr., Surf. Sci. Rep. 6, 253 (1986).

[3] Y. Jugnet, G. Grenet, T.M. Duc, in: Handbook on Synchrotron Radiation, Ed. G.V. Marr, Vol. 2, North-Holland, Amsterdam 1987, p. 663.

[4] A. Flodström, R. Nyholm, B. Johansson, in: Synchrotron Radiation Research: Advances in Surface and Interface Science, Ed. R.Z. Bachrach, Vol. 1, Plenum Press, New York 1992, p. 199.

[5] H. Petersen, Nucl. Instr. Meth. A 246, 260 (1986); E. Weschke, C. Laubschat, T. Simmons, M. Domke, G. Kaindl, Synchrotron Radiation News 4, No. 3,18 (1991).

[6] J.N. Andersen, O. Björneholm, A. Sandell, R. Nyholm, J. Forsell, L. Thånell, A. Nilsson, N. Mårtensson, Synchrotron Radiation News 4, No. 4, 21 (1991).

[7] C.T. Chen, F. Sette, Rev. Sci. Instr. 60, 1616 (1989). 
[8] K.J. Randall, J. Feldhaus, W. Erlebach, A.M. Bradshaw, W. Eberhardt, Z. Xu, Y. Ma, P.D. Johnson, Synchrotron Radiation News 4, No. 6, 16 (1991).

[9] D. Thomas, M. Coville, R. Thissen, P. Morin, Synchrotron Radiation News 5, No. 3, 8 (1992).

[10] B. Johansson, N. Mårtensson, Phys. Rev. B 21, 4427 (1980).

[11] W. Eberhardt, G. Kalkoffen, C. Kunz, Solid State Commun. 32, 901 (1979).

[12] T.-C. Chiang, D. Eastman, Phys. Rev. B 23, 6836 (1981).

[13] R. Kammerer, J. Barth, F. Gerken, C. Kunz, S.A. Flodström, L.I. Johansson, Phys. Rev. B 26, 3491 (1982).

[14] R. Nyholm, J.N. Andersen, J.F. van Acker, M. Qvarford, Phys. Rev. B 44, 10987 (1991).

[15] B. Johansson, N. Mårtensson, Helv. Phys. Acta 56, 405 (1983).

[16] P.J. Feibelman, Phys. Rev. B 39, 4866 (1989).

[17] R. Nyholm, M. Qvarford, J.N. Andersen, S.L. Sorensen, C. Wigren, J. Phys., Condens. Matter 4, 277 (1992).

[18] E. Lundgren, J.N. Andersen, M. Qvarford, R. Nyholm, submitted to Surf. Sci..

[19] N. Mårtensson, A. Stenborg, O. Björneholm, A. Nilsson, J.N. Andersen, Phys. Rev. Lett. 60, 1731 (1989).

[20] J. Gerkema, A.R. Miedema, Surf. Sci. 124, 351 (1983).

[21] Physics and Chemistry of Alkali Metal Adsorption, Eds. H.P. Bonzel, A.M. Bradshaw, G. Ertl, Elsevier, Amsterdam 1989.

[22] A. Schmalz, S. Aminpirooz, L. Becker, J. Haase, J. Neugebauer, M. Scheffler, D.R. Batchelor, D.L. Adams, E. Bögh, Phys. Rev. Lett. 67, 2163 (1991).

[23] J.N. Andersen, M. Qvarford, R. Nyholm, J.F. van Acker, E. Lundgren, Phys. Rev. Lett. 68, 94 (1992).

[24] A. Hohlfeld, K. Horn, Surf. Sci. 211/212, 844 (1989).

[25] J.N. Andersen, M. Qvarford, R. Nyholm, S.L. Sorensen, C. Wigren, Phys. Rev. Lett. 67, 2822 (1991).

[26] J.P. Biberian, M.A. van Hove, Surf. Sci. 118, 443 (1982).

[27] J.C. Tracy, P.W. Palmberg, J. Chem. Phys. 51, 4852 (1969).

[28] P. Uvdal, P.-A. Karlsson, C. Nyberg, S. Andersson, N.V. Richardson, Surf. Sci. 202, 167 (1988).

[29] H. Antonsson, A. Nilsson, N. Mårtensson, I. Panas, P.E.M. Siegbahn, J. Electron Spectrosc. Relat. Phenom. 54/55, 601 (1990). 